Article

\title{
Determining Risk Factors for the Development of Temporomandibular Disorders during Orthodontic Treatment
}

\author{
Alessandro Ugolini ${ }^{1} * * \mathbb{C}$, Federico Garbarino ${ }^{1}$, Luca Di Vece ${ }^{2}{ }^{(\mathbb{C}}$, Francesca Silvestrini-Biavati ${ }^{1}$ \\ and Valentina Lanteri ${ }^{3}(\mathbb{D}$ \\ 1 Department of Surgical and Integrated Diagnostic Sciences (DISC), University of Genova, 16132 Genoa, Italy; \\ federico.garbarino@unige.it (F.G.); francesca.silvestrini@unige.it (F.S.-B.) \\ 2 Department of Medical Biotechnologies, University of Siena, 53100 Siena, Italy; divece@unisi.it \\ 3 Department of Orthodontics Fondazione IRCCS Cà Granda, University of Milan, 20122 Milan, Italy; \\ valentina.lanteri@unimi.it \\ * Correspondence: alessandro.ugolini@unige.it; Tel.: +39-010-3538404; Fax: +39-010-3537584
}

Received: 30 October 2020; Accepted: 17 November 2020; Published: 20 November 2020

Featured Application: Authors are encouraged to provide a concise description of the specific application or a potential application of the work. This section is not mandatory.

\begin{abstract}
Temporomandibular disorders (TMD) represent a complex disease with a multifactorial etiology. Despite several studies on the subject, a causal relationship between orthodontic treatment and different forms of TMD has not been established. The aim of this study was to analyze the effect of orthodontic treatment on two aspects of TMD: myofascial pain and disc displacement. This retrospective cohort study followed 224 orthodontic adult patients at three points in time: before treatment (T0), immediately after treatment (T1), and one year after treatment (T2). Disc displacement and myofascial pain were evaluated through a clinical assessment and with a semi-structured interview, along with headache, neck, and shoulder pain parameters and behavioral and somatic accompanying symptoms. Multivariate logistic regression was used to identify risk factors that could influence the development of TMD in these patients. There was a non-significant increase in disc displacement during orthodontic treatment, which mostly resolved after completion of treatment. Myofascial pain scores worsened during treatment, but improved when compared with the baseline once treatment was complete $(\mathrm{T} 0=51.3 \%, \mathrm{~T} 1=64.6 \%, \mathrm{~T} 2=44.9 \%)$. Female gender $(\mathrm{aOR}=1.9$, CI 95\%, 1.23-2.36), the presence of somatic symptoms ( $\mathrm{aOR}=3.6$, CI 95\%, 2.01-5.84), and symptoms of anxiety or depression ( $\mathrm{aOR}=2.2, \mathrm{CI} 95 \%, 1.14-4.51)$ were significant risk factors associated with the development of TMD. There is a low and not significant risk of TMD development during orthodontic treatment. When TMD occurred, they resolved within 1 year of the end of treatment.
\end{abstract}

Keywords: occlusion; orthodontics; temporomandibular disorders; anxiety; depression

\section{Introduction}

The term TMD (temporomandibular disorders) encompasses a spectrum of disorders that are associated with pain in the masticatory muscles, temporomandibular joint, and associated structures [1]. TMD has multifactorial etiology, with anatomical, physiological, social, and psychological factors playing a role in its development [2]. Dental conditions that alter occlusion and masticatory patterns, such as trauma or chronic periodontitis, have been shown to contribute to temporomandibular joint pain [3-8]. Because of the effect that it has on occlusion, orthodontic treatment has emerged over the years as a significant factor that might contribute to the development of TMD. Several authors 
have attempted to explore the relationship between orthodontic treatment and TMD. Theoretically, orthodontic treatment is believed to upset the delicate balance of the stomatognathic system, because it has been postulated that the alteration of the occlusal morphology and the application of orthodontic forces can cause displacement of the condyle [9]. Some studies have shown that undergoing orthodontic treatment significantly increased the odds of developing signs and symptoms of TMD [10]. However, this association has not been verified by systematic reviews [11-13]. In their systematic review, Mohlin et al. [12] could not identify any clinically significant association between orthodontic treatment and TMD. More recently, Fernández-González et al. [13] performed a systematic review that explored the association between orthodontic treatment and TMD. They found that the included trials reported widely discrepant results. They concluded that there was no evidence of a cause-effect relationship between orthodontic treatment and TMD. The main drawback in several previous studies was the age group of the patients. Traditionally, orthodontic multibracket treatment is carried out in the adolescent period or in young adults. In general, the prevalence of TMD is high in this group, and the addition of orthodontic treatment could merely be a chance finding [14]. However, the demand for orthodontic treatment in adult patients is increasing and so, through a cohort of adult patients, the weight of age as a confounding factor can be resized. Another drawback was that the specific kind of TMD had not been characterized in previous studies. Myofascial pain and disc displacement are two distinct entities of TMD and may require different forms of diagnosis and management.

To this end, the aim of this medium-term retrospective study was to analyze the effect of orthodontic treatment on signs and symptoms of temporomandibular disorders in a cohort of young adult patients.

\section{Materials and Methods}

The sample was comprised of 224 adult patients seeking orthodontic treatment in two private practices from 2008 to 2018. Patients had Angle Class I, Class II, or Class III malocclusion, presented cervical vertebral maturation Stages 5 or 6, were scheduled for straight-wire orthodontic appliance therapy, and completed their TMD evaluation before treatment (T0), immediately after treatment (T1), and one year after treatment (T2). Patients who had untreated dental caries (cavitated lesions), periodontal diseases (periodontal pockets $>3 \mathrm{~mm}$ ), the presence of TMJ sounds (clicking or tripping noises), craniofacial syndromes, or systemic diseases at T0 were excluded. Patients who were below 18 years of age were also excluded from the study. The multibracket appliance treatment consisted of stainless steel brackets and molar tubes in the maxillary and mandibular arch (0.022 inch MBT prescription). A sequence of nickel titanium ( 0.012 inches and 0.016 inches) and stainless steel wires (from $0.016 \times 0.022$ inches to $0.019 \times 0.025$ inches) were used.

Demographic data, including age and gender, were recorded, and a complete medical history was obtained for all patients. Any history of facial pain, anxiety, or depression was also recorded. Details obtained during clinical examination included the type of malocclusion present (according to Angle's classification), amount of overjet and overbite, and presence of tongue thrust and crossbite. Oral hygiene status was also evaluated using the Silness and Loe plaque index [15].

All clinical procedures on humans described in the present manuscript were carried out in accordance with national law and the Helsinki Declaration of 1975 in its current revised form. The data were collected retrospectively. The experimental study was approved by the Regional Ethics Committees (Prot. N. 355-14). All patients signed written, informed consent, and they also signed written permission to use their medical records for retrospective studies.

\section{Evaluation for TMD}

Prior to beginning fixed orthodontic therapy, each patient was routinely evaluated for signs and symptoms of TMD. Clinical assessment was carried out by a senior calibration (A.U.) examiner and was based on the parameters given in the RDC/TMD criteria [16]. The two characteristics-assessment of myofascial pain and assessment of disc dysfunction—were assessed separately. 
Assessment of myofascial pain was done using the modified pericranial tenderness score (PTS), described in previous studies [17-21]. This included palpation of the masticatory muscles (masseter and temporalis muscles) and cervical muscles (sternocleidomastoid, trapezius, and suboccipital muscles). We used a pressure-controlled palpation technique described by Bendtsen et al. [22]. Palpation was done using the index and middle fingers; small circular movements were carried out for the masticatory and suboccipital muscles, while the other cervical muscles were gently pinched. Each muscle was palpated for at least three seconds. The tenderness on palpation at each muscle was graded from 0 to 3, with 0 indicating normal tone and 1, 2, and 3 indicating mild, moderate, and severe tenderness, respectively. The final PTS score was divided into two components: the masticatory muscle tenderness score (MTS) for the first group of muscles and the cervical muscle tenderness score (CTS) for the second group. Both the MTS and CTS were calculated as the mean for the scores of each muscle in that group $[18,21]$.

A calibration session was performed every 2 years with a second senior examiner to assess the degree of the inter-rating agreement between clinicians. The results were found to have acceptable reliability (the $\mathrm{K}$ value for combined intraclass correlation for within-subject variability and between-subject variability was $0.92,95 \%$ CI, $0.89-0.96, p<0.001$ ) that was consistent with previous reports [21].

To assess for disc displacement, two components were considered. The presence of clicking was assessed through direct examination (by placing the operator's fingers over the joint region), as well as by auscultation. The presence or absence of clicking was recorded during opening and closing movements. The range of motion of the joint was assessed by checking the degree of mouth opening and opening patterns.

The evaluation methods for TMD were repeated at the baseline and at the end of treatment, and they were continued until one year after the fixed appliance was removed. According to orthodontic clinical protocol, the development of TMD symptoms was routinely evaluated at the baseline before treatment (T0), immediately after treatment (T1), and one year after treatment (T2). A psychological assessment, according to Axis 1 of the Diagnostic and Statistical Manual of Mental Disorders, 4th Edition, was then carried out at the baseline before the start of orthodontic treatment. The presence of anxiety disorders and depression was investigated with an SCID-I-structured, semi-structured clinical interview [22], along with headache, neck, and shoulder pain parameters and somatic symptoms (colitis, gastritis, swallowing or digestion difficulty, phobias, tinnitus, dizziness, cramps, paresthesia, back pain, headache, and oral parafunction) [18-23]. The SCID-I assessment was carried out by a clinician with 15 years of experience in treating TMD patients, calibrated for SCID analysis by a senior psychologist of the local headache and orofacial pain center.

The primary outcome parameter studied was the development of TMD during and one year after orthodontic treatment. The influence of multiple risk factors (including demographic and clinical data) on the development of TMD was also assessed using multivariate logistic regression analysis. The ORs were adjusted for age, sex, and presence of anxiety or mood disorders. The significance of the analysis was evaluated by setting the $p$ value to less than 0.05 .

\section{Results}

A total of 224 patients were selected for this study. Of these, 105 patients were male and 119 were female. The mean age of the patients at the time of beginning orthodontic treatment was $28 \pm 8.3$ years (Table 1).

On clinical examination, it was found that 116 patients (51.9\%) had Class I malocclusions. 87 patients (38.6\%) presented for the correction of Class II malocclusions, and 21 patients $(9.5 \%)$ presented for the correction of Class III malocclusions. 94 patients $(41.1 \%)$ were affected by tongue thrust, and 44 patients presented crossbite (19.6\%). More than half of the patients $(52.5 \%)$ had compromised oral hygiene statuses prior to beginning treatment. Forty patients $(17.7 \%)$ required the 
extraction of premolars during treatment. The average duration of treatment was $21 \pm 3.5$ months (Table 1). All patients were followed up on for at least one year after treatment.

Table 1. Characteristics of the sample. MTS = masticatory muscle tenderness score; CTS = cervical muscle tenderness score; $\mathrm{T} 0=$ before treatment; $\mathrm{T} 1=$ immediately after treatment; $\mathrm{T} 2=$ one year after treatment; $\mathrm{SD}=$ standard deviation.

\begin{tabular}{|c|c|c|c|c|}
\hline \multirow[t]{2}{*}{ Sample $n^{\circ}=224$} & \multicolumn{4}{|c|}{ Subjects } \\
\hline & Males & Females & Total & $\%$ \\
\hline Gender & 105 & 119 & 224 & \\
\hline Age at $\mathrm{T} 0$ (mean $\pm \mathrm{SD}$ in years) & \multicolumn{2}{|c|}{$28 \pm 8.3$} & & \\
\hline Anxiety or depression at T0 & 18 & 39 & 57 & 25.4 \\
\hline Somatic symptoms $>4$ & 31 & 55 & 86 & 38.3 \\
\hline Class I malocclusion at T0 & 55 & 61 & 116 & 51.9 \\
\hline Class II malocclusion at T0 & 37 & 50 & 87 & 38.6 \\
\hline Class III malocclusion at T0 & 14 & 7 & 21 & 9.5 \\
\hline Extraction of premolars during treatment & 17 & 23 & 40 & 17.7 \\
\hline Duration of orthodontic treatment (T1 - T0 in months) & \multicolumn{3}{|c|}{$21 \pm 3.5$} & \\
\hline Overjet $(\mathrm{mm})$ at $\mathrm{T} 0$ & \multicolumn{3}{|c|}{$5 \pm 4.1$} & \\
\hline Overbite $(\mathrm{mm})$ at T0 & \multicolumn{3}{|c|}{$4 \pm 3.9$} & \\
\hline Tongue thrust at T0 & 49 & 45 & 94 & 42.1 \\
\hline $\begin{array}{l}\text { Oral hygiene status during treatment (plaque index } \\
\qquad \text { Silness/Loe }>2 \text { ) [15] }\end{array}$ & 64 & 54 & 118 & 52.5 \\
\hline Crossbite at T0 & 20 & 24 & 44 & \\
\hline \multicolumn{5}{|l|}{ TMD evaluation } \\
\hline & Males & Females & Total & $\%$ \\
\hline Clicking of the TMJ at T0 & \multicolumn{4}{|c|}{ Symptom free at $\mathrm{T} 0$ (selection criteria) } \\
\hline Clicking of the TMJ at T1 & 9 & 18 & 27 & 12.0 \\
\hline Clicking of the TMJ at T2 & 3 & 7 & 10 & 4.4 \\
\hline MTS score $\geq 2$ at T0 & 45 & 70 & 115 & 51.3 \\
\hline MTS score $\geq 2$ at T1 & 55 & 89 & 145 & 64.6 \\
\hline MTS score $\geq 2$ at T2 & 41 & 60 & 101 & 44.9 \\
\hline CTS score $\geq 2$ at T0 & 62 & 78 & 141 & 62.7 \\
\hline CTS score $\geq 2$ at $\mathrm{T} 1$ & 67 & 88 & 155 & 69.0 \\
\hline CTS score $\geq 2$ at T2 & 55 & 72 & 127 & 56.9 \\
\hline
\end{tabular}

\section{Development of Symptoms of TMD}

None of the patients had clicking at T0, as this was one of the selection criteria. At T1, 27 patients $(12 \%)$ developed clicking. However, one year after treatment as completed, only 10 patients $(4.4 \%)$ continued to have clicking at T2 (Table 1).

Prior to beginning orthodontic treatment, 115 patients (51.2\%) had tenderness in their masticatory muscles, and 141 patients experienced tenderness in their cervical muscles $(62.6 \%)$. This value actually increased during the course of treatment, and at T1, 145 patients had tenderness in their masticatory muscles $(64.5 \%)$ and 155 had tenderness in their cervical muscles $(69 \%)$. However, one year after completion of orthodontic treatment, the number of patients with masticatory muscle tenderness dropped to 101 (44.9\%), while the number of patients with cervical muscle tenderness dropped to 127 $(56.9 \%)$ at T2. Overall, the incidence of myofascial pain dropped from $56.9 \%$ before treatment to $52.9 \%$ after treatment (Table 1).

The influence of multiple risk factors on the odds of developing TMD is highlighted in Table 1. It was clearly seen, even after ORs were adjusted for age, sex, and the presence of anxiety or mood disorders, that gender played a crucial role in the development of TMD. On average, women had $90 \%$ higher odds than men of developing TMD (Table 1, Figure 1). 


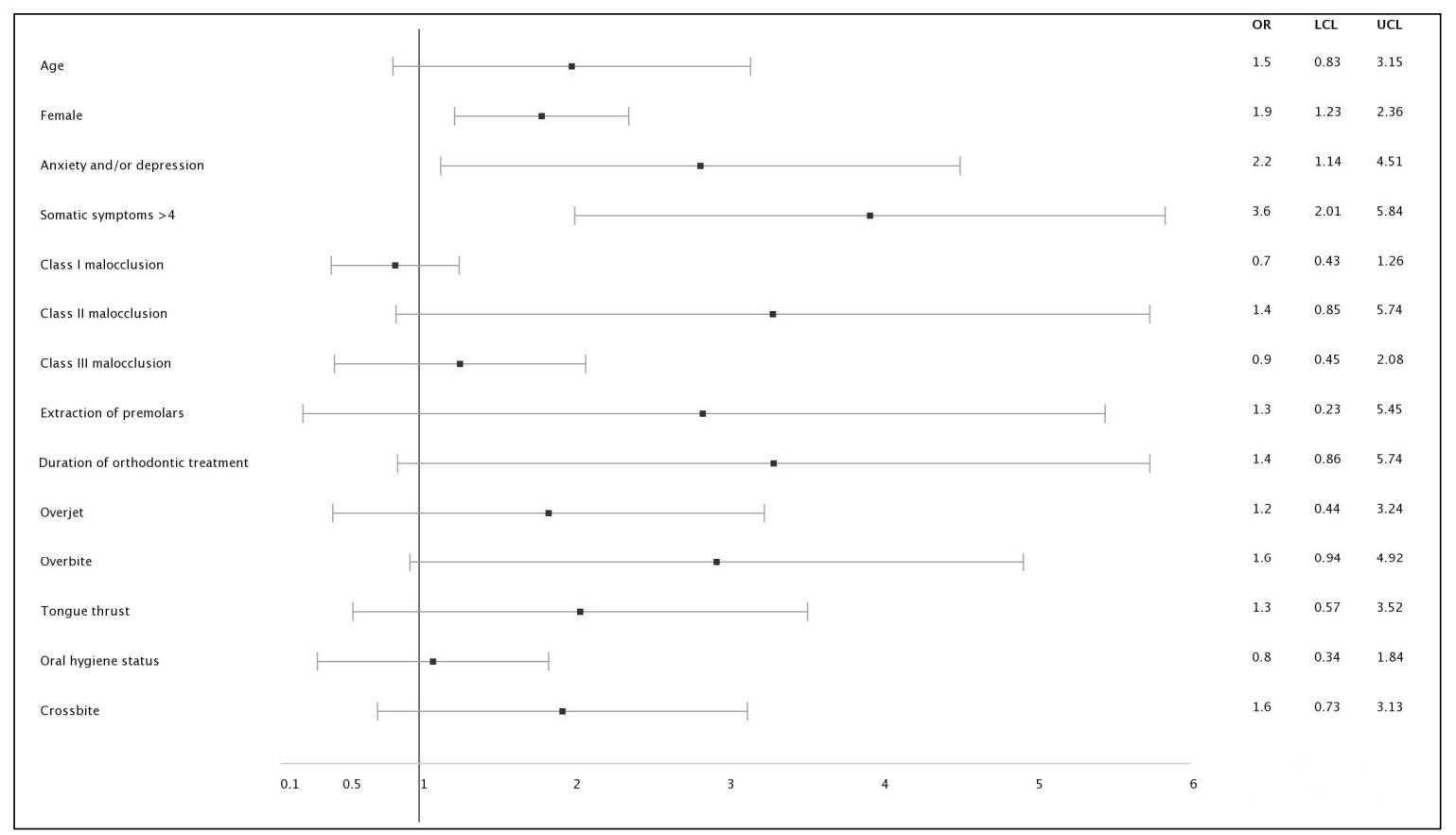

Figure 1. Forest plot of multivariate analysis. OR = odds ratio; $\mathrm{IC}=95 \%$ confidence interval. $\mathrm{LCL}=$ 95\% lower confidence limit; UCL $=95 \%$ upper confidence limit.

The presence of accompanying symptoms, both behavioral and somatic, prior to orthodontic treatment also influenced the development of TMD. In the study, $25.4 \%$ of patients reported symptoms of anxiety or depression prior to the beginning of orthodontic treatment. Moreover, at T0, 38.3\% of the sample suffered from more than four somatic symptoms (occurring at least monthly), as was self-reported by the patients during the clinical interview. It was found that patients who reported these symptoms had 2.2 (anxiety or depression) and 3.6 (somatic symptoms) times greater odds of developing TMD. Other factors, including the type of malocclusion and the duration of orthodontic treatment, did not show any evidence of modulating the risk of developing TMD (Table 2, Figure 1).

Table 2. Logistic regression analysis of the risk of various factors in the development of temporomandibular disorders (TMD) following orthodontic treatment. The ORs were adjusted for age, gender, and the presence of anxiety or mood disorders. $95 \% \mathrm{CI}=95 \%$ confidence interval. $*=p$ values $<0.01$.

\begin{tabular}{cccccc}
\hline Variable & $\begin{array}{c}\boldsymbol{\beta} \text { (Regression } \\
\text { Coefficient) }\end{array}$ & Odds Ratio & 95\% CI & $p$ Value \\
\hline Age & 0.581 & 1.5 & 0.83 & 3.15 & 0.28 \\
Female & 1243 & 1.9 & 1.23 & 2.36 & $<0.01^{*}$ \\
Anxiety or depression & 1.398 & 2.2 & 1.14 & 4.51 & $<0.01^{*}$ \\
Somatic symptoms $>4$ & 2048 & 3.6 & 2.01 & 5.84 & $<0.01^{*}$ \\
Class I malocclusion & -0.326 & 0.7 & 0.43 & 1.26 & 0.76 \\
Class II malocclusion & -0.277 & 1.4 & 0.85 & 5.74 & 0.14 \\
Class III malocclusion & 0.438 & 0.9 & 0.45 & 2.08 & 0.86 \\
Extraction of premolars & 0.627 & 1.3 & 0.23 & 5.45 & 0.74 \\
Duration of orthodontic & -0.562 & 1.4 & 0.86 & 5.74 & 0.14 \\
treatment & 0.273 & 1.2 & 0.44 & 3.24 & 0.31 \\
Overjet & -0.364 & 1.6 & 0.94 & 4.92 & 0.14 \\
Overbite & 0.442 & 1.3 & 0.57 & 3.52 & 0.32 \\
Tongue thrust & -0.383 & 0.8 & 0.34 & 1.84 & 0.42 \\
Oral hygiene status & 0.397 & 1.6 & 0.73 & 3.13 & 0.31 \\
Crossbite & & & & & \\
\hline
\end{tabular}




\section{Discussion}

This was a cohort study that attempted to ascertain the incidence of TMD at two different time points as compared with the baseline: during orthodontic treatment and one year after orthodontic treatment. Results showed that patients developed a low and not statistically significant increase in disc displacement, and there was a reduction in myofascial pain as compared with the baseline. There is not much evidence in the literature linking the development of disc displacement with orthodontic treatment. Literature reviews have shown that all forms of abnormal occlusion, including exaggerated Class II or Class III occlusions, edge-to-edge bite, or increased or reduced overjet and overbite, could increase the risk of developing disc displacement [24]. It is possible that, during the course of orthodontic treatment, the abnormal occlusion that develops before the bite is settled could bring on forward movement of the articular disc. In most cases, this appears to be temporary, and the clicking resolves on its own. However, in some cases, it could persist. In our study, all cases of disc displacement were merely associated with asymptomatic clicking, and we did not encounter cases that progressed to painful clicking or non-reducing displacements one year after the end of orthodontic treatment.

Myofascial pain, although considered under TMD diagnoses, is a different entity. Several patients suffer from mild myofascial pain without even being aware of their condition. In the current study, on clinical examination, we found that more than half of the patients were already experiencing muscle tenderness. In a prospective case control study, Tecco et al. [25] assessed the effect of fixed orthodontic treatment on myofascial pain. They found that orthodontic treatment helped to improve the signs and symptoms of myofascial pain, although complete resolution did not occur. In the current study, we also found that there was a slight decrease in the incidence of muscle tenderness as compared with the baseline.

In the current study, to assess myofascial pain, we used the modified pericranial muscle tenderness score described by Aaseth et al. [17]. This score was originally developed for use with tension headaches. However, the two conditions are not unrelated, and this technique would be equally effective in detecting myofascial pain secondary to TMD [18]. The MTS and CTS scores evaluated two groups of muscles: the masticatory muscles and the cervical muscles. Cervical muscle tenderness is an important component of TMD. Almoznino et al. [26] showed that cervical muscle tenderness was a prominent feature in patients with myogenous TMD, but only Tecco et al. [25] have evaluated pain in the cervical muscles during orthodontic treatment, reporting an improvement in myofascial pain after orthodontic treatment. In the current study, when only cervical tenderness was taken into account, it was found that the tenderness score actually decreased when compared with the baseline one year after orthodontic treatment. This parameter, therefore, is one that deserves more consideration in future studies.

Several studies have tried to establish risk factors for developing TMD following orthodontic treatment [11-13]. Age has been demonstrated as a risk factor for development of TMD in previous studies. In a longitudinal study, McFarlane et al. [27] found that young adults and adolescents, especially between the ages of 19 and 20, demonstrated a higher risk of persistent TMD following orthodontic treatment. In the current study, we found that a one unit increase in age increased the odds of developing TMD by $50 \%$. However, these results were not statistically significant. Previous studies have highlighted that the female gender is more susceptible to the development of TMD following orthodontic treatment. This study showed that women had almost double the odds of developing TMD compared with men. The study by McFarlane et al. [27] demonstrated slightly higher odds in a three-year follow-up period ( $\mathrm{OR}=2.45$ ). However, this decreased during further follow-up visits.

Does the type of malocclusion have some effect on the development of temporomandibular disorders? In the current study, Class II malocclusions had increased odds of developing TMD, while Class I had the least odds. These results, however, were not statistically significant. Previous studies have shown weak evidence that Class II malocclusions are associated with TMD [28].

Major risk factors identified for TMD development were the presence of more than four somatic symptoms occurring at least monthly and the presence of anxiety or depression at the baseline. In the 
current study, this tripled the odds of developing TMD during orthodontic treatment. Previous studies have shown that TMD patients have higher anxiety levels compared with normal patients, but a cause-effect relationship between anxiety or depression and TMD has yet to be established [18,29-32].

Anxiety and depressive initial symptoms, along with the somatic accompanying symptoms, may promote muscular hyperactivity and altered muscle mechanics that may lead to muscle pain $[29,33]$. In the craniofacial area, muscle disorders may initiate TMJ inflammation and pain. These are related to abnormal pain processing in the trigeminal system, which involves components of the neurotransmitter system (serotonin and catecholamines) [29,33-35].

Recently, the involvement of catecholamine O-methyl transferase (COMT), which is implicated in the regulation of pain sensitivity genes, has been also reported [36,37]. Therefore, TMD pain may reflect the physical manifestation of depression and anxiety, even though a direct relationship has not been demonstrated.

One of the limitations of this study is the lack of radiological diagnosis due to the difficulty in performing magnetic resonance imaging (MRI) during orthodontic treatment. The diagnosis of TMJ dysfunction may not be determined by clinical examination alone, but the diagnosis is carried out by a combination of clinical manifestations and diagnostic imaging confirmation. Above all, an MRI is helpful when clinical examination cannot predict disk position. Disk positional modifications have been considered one of the main risk factors related to the development of abnormal mechanical trauma and inflammatory disorders within temporomandibular disorders. However, at the end of treatment, an MRI was performed on all symptomatic patients and, in 100\% of the cases, the clinical diagnosis was confirmed.

Moreover, retrospective studies are susceptible to selection bias due to their design and are also associated with the deflation of treatment effects. In this research, to reduce selection bias, a precise case definition was set by several clinical and morphological variables. This process, together with the large sample size, produced a study group that reflected the exposure of the population that gave rise to the cases and minimized the selection bias.

In view of the findings of the current study, the following recommendations can be made. Firstly, each patient who is being considered for orthodontic treatment must be evaluated for temporomandibular joint dysfunction, and this must be documented if present. If the patient has any modifiable risk factors, such as behavioral or somatic accompanying symptoms, an attempt must be made to improve or correct them. Prior to beginning orthodontic treatment, the risk of developing temporomandibular joint dysfunction must be explained to the patients as part of their informed consent. Finally, at each orthodontic appointment, the clinician must ascertain whether the patient has experienced symptoms of TMD and, if they have, must examine the TMJ for any dysfunction. The results of the current study show that most cases of TMD related to orthodontic treatment are temporary and resolve after the completion of the treatment.

In conclusion, this study found a slight increase in TMD signs and symptoms during the course of orthodontic treatment, which were mostly resolved upon completion of treatment. There was an actual improvement in myofascial pain after orthodontic treatment.

Author Contributions: A.U. contributed to the design of the study, analysis and interpretation of data, and drafted the manuscript; F.G. contributed to the acquisition and interpretation of data and critically revised the manuscript; L.D.V. contributed to the interpretation of data; F.S.-B. contributed to the conception and design of the study and critically revised the manuscript; V.L. contributed to the acquisition and interpretation of data and critically revised the manuscript. All authors have read and agreed to the published version of the manuscript.

Funding: This research received no external funding.

Conflicts of Interest: The authors declare no conflict of interest.

\section{References}

1. Ohrbach, R.; Dworkin, S.F. The Evolution of TMD Diagnosis: Past, Present, Future. J. Dent. Res. 2016, 95, 1093-1101. [CrossRef] [PubMed] 
2. Liu, F.; Steinkeler, A. Epidemiology, diagnosis, and treatment of temporomandibular disorders. Dent. Clin. 2013, 57, 465-479. [CrossRef] [PubMed]

3. Jeon, H.M.; Ahn, Y.W.; Jeong, S.H.; Ok, S.M.; Choi, J.; Lee, J.Y.; Joo, J.Y.; Kwon, E.Y. Pattern analysis of patients with temporomandibular disorders resulting from unilateral mastication due to chronic periodontitis. J. Periodontal Implant Sci. 2017, 47, 211-218. [CrossRef] [PubMed]

4. Ugolini, A.; Parodi, G.B.; Casali, C.; Silvestrini-Biavati, A.; Giacinti, F. Work-related traumatic dental injuries: Prevalence, characteristics and risk factors. Dent. Traumatol. 2018, 34, 36-40. [CrossRef]

5. Lobbezoo, F.; Ahlberg, J.; Raphael, K.G.; Wetselaar, P.; Glaros, A.G.; Kato, T.; Santiago, V.; Winocur, E.; De Laat, A.; De Leeuw, R.; et al. International consensus on the assessment of bruxism: Report of a work in progress. J. Oral Rehabil. 2018, 45, 837-844. [CrossRef]

6. Al-Baghdadi, M.; Durham, J.; Araujo-Soares, V.; Robalino, S.; Errington, L.; Steele, J. TMJ Disc Displacement without Reduction Management: A Systematic Review. J. Dent. Res. 2014, 93, 37S-51S. [CrossRef]

7. Mongini, F.; Evangelista, A.; Rota, E.; Ferrero, L.; Ugolini, A.; Ceccarelli, M.; Ciccone, G.; Galassi, C. Long-term benefits of an educational and physical program on headache, and neck and shoulder pain, in a working community. J. Pain 2009, 10, 1138-1145. [CrossRef]

8. Mongini, F.; Evangelista, A.; Rota, E.; Ferrero, L.; Ugolini, A.; Milani, C.; Ceccarelli, M.; Joffrain, L.; Ciccone, G.; Galassi, C. Further evidence of the positive effects of an educational and physical program on headache, neck and shoulder pain in a working community. J. Headache Pain 2010, 11, 409-415. [CrossRef]

9. Conti, A.C.; Oltramari, P.V.; Navarro, R.D.; Almeida, M.R. Examination of temporomandibular disorders in the orthodontic patient: A clinical guide. J. Appl. Oral Sci. 2007, 15, 77-82. [CrossRef]

10. Hwang, S.H.; Park, S.G. Experience of Orthodontic Treatment and Symptoms of Temporomandibular Joint in South Korean Adults. Iran J. Public Health 2018, 47, 13-17.

11. Michelotti, A.; Iodice, G. The role of orthodontics in temporomandibular disorders. J. Oral Rehabil. 2010, 37, 411-429. [CrossRef] [PubMed]

12. Mohlin, B.; Axelsson, S.; Paulin, G.; Pietilä, T.; Bondemark, L.; Brattström, V.; Hansen, K.; Holm, A.K. TMD in relation to malocclusion and orthodontic treatment: A systematic review. Angle Orthod. 2007, 77, 542-548. [CrossRef]

13. Fernández-González, F.J.; Cañigral, A.; López-Caballo, J.L.; Brizuela, A.; Moreno-Hay, I.; del Río-Highsmith, J.; Vega, J.A. Influence of orthodontic treatment on temporomandibular disorders. A systematic review. J. Clin. Exp. Dent. 2015, 7, e320. [CrossRef] [PubMed]

14. Minghelli, B.; Cardoso, I.; Porfírio, M.; Gonçalves, R.; Cascalheiro, S.; Barreto, V.; Soeiro, A.; Almeida, L. Prevalence of temporomandibular disorder in children and adolescents from public schools in Southern Portugal. N. Am. J. Med. Sci. 2014, 6, 126-132. [CrossRef]

15. Fischman, S.L. Current status of indices of plaque. J. Clin. Periodontol. 1986, 13, 371-374. [CrossRef]

16. Schiffman, E.; Ohrbach, R.; Truelove, E.; Look, J.; Anderson, G.; Goulet, J.P.; List, T.; Svensson, P.; Gonzalez, Y.; Lobbezoo, F.; et al. Diagnostic Criteria for Temporomandibular Disorders (DC/TMD) for Clinical and Research Applications: Recommendations of the International RDC/TMD Consortium Network and Orofacial Pain Special Interest Group. J. Oral Facial Pain Headache 2014, 28, 6-27. [CrossRef] [PubMed]

17. Aaseth, K.; Grande, R.B.; Lundqvist, C.; Russell, M.B. Pericranial tenderness in chronic tension-type headache: The Akershus population-based study of chronic headache. J. Headache Pain 2014, 15, 58. [CrossRef]

18. Mongini, F.; Ciccone, G.; Ceccarelli, M.; Baldi, I.; Ferrero, L. Muscle tenderness in different types of facial pain and its relation to anxiety and depression: A cross-sectional study on 649 patients. Pain 2007, 131, 106-111. [CrossRef]

19. Jensen, R.; Rasmussen, B.K. Muscular disorders in tension-type headache. Cephalalgia 1996, 16, 97-103. [CrossRef]

20. Hatch, J.P.; Rugh, J.D.; Sakai, S.; Prihoda, T.J. Reliability of the craniomandibular index. J. Orofac. Pain 2002, 16, 284-295.

21. Pezzoli, M.; Ugolini, A.; Rota, E.; Ferrero, L.; Milani, C.; Pezzoli, L.; Pecorari, G.; Mongini, F. Tinnitus and its relationship with muscle tenderness in patients with headache and facial pain. J. Laryngol. Otol. 2015, 129, 638-643. [CrossRef]

22. Mazzi, F.; Morosini, P.; De Girolamo, G.; Lussetti, M.; Guaraldi, G.P. SCID-I-Structured Clinical Interview for DSM-IV Axis I Disorders, Italian Edition; OS Organizzazioni Speciali: Firenze, Italy, 2000. 
23. Bendtsen, L.; Jensen, R.; Jensen, N.K.; Olesen, J. Pressure-controlled palpation: A new technique which increases the reliability of manual palpation. Cephalalgia 1995, 15, 205-210. [CrossRef]

24. Chisnoiu, A.M.; Picos, A.M.; Popa, S.; Chisnoiu, P.D.; Lascu, L.; Picos, A.; Chisnoiu, R. Factors involved in the etiology of temporomandibular disorders-A literature review. Clujul Med. 2015, 88, 473-478. [CrossRef] [PubMed]

25. Tecco, S.; Marzo, G.; Crincoli, V.; Di Bisceglie, B.; Tetè, S.; Festa, F. The prognosis of myofascial pain syndrome (MPS) during a fixed orthodontic treatment. Cranio 2012, 30, 52-71. [CrossRef] [PubMed]

26. Almoznino, G.; Zini, A.; Zakuto, A.; Zlutsky, H.; Bekker, S.; Shay, B.; Haviv, Y.; Sharav, Y.; Benoliel, R. Cervical muscle tenderness in temporomandibular disorders and its association with diagnosis, disease related outcomes, and comorbid pain conditions. J. Oral Facial Pain Headache. 2020, 34, 67-76. [CrossRef] [PubMed]

27. MacFarlane, T.V.; Kenealy, P.; Kingdon, H.A.; Mohlin, B.O.; Pilley, J.R.; Richmond, S.; Shaw, W.C. Twenty-year cohort study of health gain from orthodontic treatment: Temporomandibular disorders. Am. J. Orthod. Dentofac. Orthop. 2009, 135, 692.e1-692.e8. [CrossRef]

28. Keeling, S.D.; McGorray, S.; Wheeler, T.T.; King, G.J. Risk factors associated with temporomandibular joint sounds in children 6 to 12 years of age. Am. J. Orthod. Dentofac. Orthop. 1994, 105, 279-287. [CrossRef]

29. Kindler, S.; Samietz, S.; Houshmand, M.; Grabe, H.J.; Bernhardt, O.; Biffar, R.; Kocher, T.; Meyer, G.; Völzke, H.; Metelmann, H.R.; et al. Depressive and anxiety symptoms as risk factors for temporomandibular joint pain: A prospective cohort study in the general population. J. Pain 2012, 13, 1188-1197. [CrossRef]

30. Mongini, F.; Rota, E.; Evangelista, A.; Ciccone, G.; Milani, C.; Ugolini, A.; Ferrero, L.; Mongini, T.; Rosato, R. Personality profiles and subjective perception of pain in head pain patients. Pain 2009, 144, 125-129. [CrossRef] [PubMed]

31. List, T.; Jensen, R.H. Temporomandibular disorders: Old ideas and new concepts. Cephalalgia 2017, 37, 692-704. [CrossRef]

32. Rota, E.; Evangelista, A.; Ciccone, G.; Ferrero, L.; Ugolini, A.; Milani, C.; Ceccarelli, M.; Galassi, C.; Mongini, F. Effectiveness of an educational and physical program in reducing accompanying symptoms in subjects with head and neck pain: A workplace controlled trial. J. Headache Pain 2011, 12, 339-345. [CrossRef] [PubMed]

33. Scrivani, S.J.; Keith, D.A.; Kaban, L.B. Temporomandibular disorders. N. Engl. J. Med. 2008, 359, $2693-2705$. [CrossRef] [PubMed]

34. Apkarian, A.V.; Baliki, M.N.; Geha, P.Y. Towards a theory of chronic pain. Prog. Neurobiol. 2009, 87, 81-97. [CrossRef] [PubMed]

35. Slade, G.D.; Ohrbach, R.; Greenspan, J.D.; Fillingim, R.B.; Bair, E.; Sanders, A.E.; Dubner, R.; Diatchenko, L.; Meloto, C.B.; Smith, S.; et al. Painful Temporomandibular Disorder: Decade of Discovery from OPPERA Studies. J. Dent. Res. 2016, 95, 1084-1092. [CrossRef]

36. Schwahn, C.; Grabe, H.J.; Meyer zu Schwabedissen, H.; Teumer, A.; Schmidt, C.O.; Brinkman, C.; Kocher, T.; Nauck, M.; Volzke, H.; Biffar, R.; et al. The effect of catechol-O-methyltransferase polymorphisms on pain is modified by depressive symptoms. Eur. J. Pain 2012, 16, 878-889. [CrossRef]

37. Michelotti, A.; Liguori, R.; Toriello, M.; D'Antò, V.; Vitale, D.; Castaldo, G.; Sacchetti, L. Catechol-O-methyltransferase (COMT) gene polymorphisms as risk factor in temporomandibular disorders patients from Southern Italy. Clin. J. Pain 2014, 30, 129-133. [CrossRef]

Publisher's Note: MDPI stays neutral with regard to jurisdictional claims in published maps and institutional affiliations.

(C) 2020 by the authors. Licensee MDPI, Basel, Switzerland. This article is an open access article distributed under the terms and conditions of the Creative Commons Attribution (CC BY) license (http://creativecommons.org/licenses/by/4.0/). 